\title{
Change in perceived spatial directions due to context
}

\author{
N. SCHOUMANS \\ Delft University of Technology, Delft, The Netherlands \\ and \\ J. J. KOENDERINK and A. M. L. KAPPERS \\ Helmholtz Institute, Utrecht, The Netherlands
}

\begin{abstract}
We examined the influence of context on exocentric pointing. In a virtual three-dimensional set-up, we asked our subjects to aim a pointer toward a target in two conditions: The target and the pointer were visible alone, or they were visible with planes through each of them. The planes consisted of a regular grid of horizontal and vertical lines. The presence of the planes had a significant influence on the indicated direction. These changes in indicated direction depended systematically on the orientation of the planes relative to the subject and on the angle between the planes. When the orientation of the (perpendicular) planes varied from asymmetrical to symmetrical to the frontoparallel plane, the indicated direction varied over a range of $15^{\circ}$-from a slightly larger slant to a smaller slant-as compared with the condition without the contextual planes. When the dihedral angle between the two planes varied from $90^{\circ}$ to $40^{\circ}$, the indicated direction varied over a range of less than $5^{\circ}$ : A smaller angle led to a slightly larger slant. The standard deviations in the indicated directions (about $3^{\circ}$ ) did not change systematically. The additional structure provided by the planes did not lead to more consistent pointing. The systematic changes in the indicated direction contradict all theories that assume that the perceived distance between any two given points is independent of whatever else is present in the visual field - that is, they contradict all theories of visual space that assume that its geometry is independent of its contents (e.g., Gilinsky, 1951; Luneburg, 1947; Wagner, 1985).
\end{abstract}

Human observers report a single, stable image of the surrounding three-dimensional (3-D) space. For the normal observer, the two frontally spaced eyes provide the main source of information for localizing objects. In everyday life, these objects are usually located on planes: A cup is placed on a table; a photograph hangs on a wall. Surprisingly, little is known about the influence, if any, of such contextual planes on spatial judgments. Few reports exist on the role of a floor (e.g., Ellis, Smith, Grunwald,

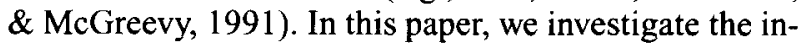
fluence of contextual planes on exocentric pointing.

Optically perceived space (or visual space, for short) is distorted with respect to physical space (see the experiments by Blumenfeld, 1913; Foley \& Richards, 1972; Indow \& Watanabe, 1984; Wagner, 1985). Luneburg (1947) provided a classical theoretical description of visual space and its relation with physical space. He assumed, among other things, that visual space is Riemannian and that the geometry of visual space is independent of its contents. Although parts of his theory have been adapted (Blank, 1961) and some of his assumptions have been questioned (Battro, di Pierro Netto, \& Rozestraten, 1975; Foley, 1964; Indow \& Watanabe, 1984), all models of visual space still assume visual space to have a metric

Correspondence concerning this article should be addressed to A. M. L. Kappers, Helmholtz Instituut, Princetonplein 5, 3584 CC Utrecht, The Netherlands (e-mail: a.m.l.kappers@phys.uu.nl). and to have a geometry independent of its contents (Blank, 1961; Foley, 1991; Gilinsky, 1951; Wagner, 1985). In other words, they assume that all perceived spatial relations depend only on the location of objects relative to the observer and do not depend on the context of those locations-that is, whatever else is in the scene. Even though Wagner states that "the geometry of visual space itself appears to be a function of stimulus conditions" (p. 493) his model does not incorporate a possible role for context.

However, certain experiments in other fields of research into visual perception have demonstrated a specific influence of context. We want to mention two examples: the depth contrast effect and the rod frame experiments. The depth contrast effect (Werner, 1938) is an effect of the orientation of the larger background plane on the perceived orientation of a smaller plane in front of it (see, e.g., Kumar \& Glaser, 1992; van Ee \& Erkelens, 1996). The rod frame experiments (Witkin \& Asch, 1948) have demonstrated that the perceived vertical depends on the orientation of the largest visible frame. Both of these types of experiments suggest that context is used as reference and suggest that context influences perceived spatial relations. However, these experiments are very different (different in focus, in experimental set-up, and in task) from those performed with the purpose of investigating visual space, and therefore, they cannot be compared directly. There are other examples, from the field of hyperacuity (Mitchison \& Westheimer, 1984) and from the study of illusions (Gogel, 1984; for the Poggendorff illu- 
sion, see, e.g., Tolansky, 1964), but these are even less related to the experiments investigating visual space.

There also have been some theoretical statements and phenomenological reports that suggest an influence of context on perceived spatial relations. Gestalt theories do emphasize that perception is only meaningful in the presence of a context (e.g., Gibson, 1950; Koffka, 1935). More specifically, Koffka states that "lines will be determined in their direction and other aspects by the things or surfaces to which they belong" (p. 215). The phenomenological reports mainly come from the arts, such as architecture, theater, and painting. There, it has been shown frequently that space perception can be influenced by adding a context with false perspective (see Pirenne, 1970, for examples, such as the Pozzo ceiling, Piazza Spada, and del Campidoglio in Rome). Within visual science, the Ames room and the trapezoid window are wellknown examples (Ames, 1953 and 1951, respectively). Gogel (Gogel, 1984; Gogel \& Mershon, 1977) has demonstrated that the suggested perspective in a trapezoid window can influence the perceived location in depth of an object close to the window. However, despite these theories and studies of illusions, there has never been a clear systematic experiment showing that context influences spatial perception.

The major aim of this study is to find out whether contextual planes have a significant influence on exocentric pointing, on either the veridicality of pointing or the consistency of the pointing. To this end, we compared exocentric pointing in conditions with and without contextual planes. We used a number of different contextual planes, because a demonstration of an influence of context would become even more convincing if the changes in exocentric pointing also varied systematically with variations in the planes.

In line with most studies of visual space, we used a few luminous objects in an otherwise dark environment. Previously (Schoumans \& Denier van der Gon, 1999), we measured an exocentric pointing task with a pointer and a target as the only luminous objects in an otherwise dark room. We found standard deviations smaller than would be expected from the resolution of the retina and from psychophysically measured depth-difference detection thresholds. In other words, such a task can be performed with high accuracy in the absence of any context.

We chose an exocentric pointing task because it is a typical spatial task that we perform in everyday life: Monitoring who looks at whom in a group of people is probably the commonest example. Studying exocentric pointing is also a good method of studying spatial relations and has been used as such before (e.g., Ellis et al., 1991; Koenderink \& van Doorn, 1998). It should be noted that any affine transformation (such as a scale, rotation, or shear), such as Wagner's (1985) model, or even a projective transformation between visual space and physical space would not result in systematic errors in pointing.
Systematic errors in exocentric pointing indicate a more complex, nonlinear distortion of visual space.

A clear demonstration of an influence of contextual planes on exocentric pointing will have immediate implications for the existing models of visual space, since most of these (such as Luneburg's, 1947, model) do not incorporate a possible influence of context into the theory. Thus, this study is designed to disprove this class of models.

\section{METHOD}

\section{Subjects}

There were 5 subjects, who had given their informed consent and were naive as to the purpose of the experiments. All the subjects had normal vision or corrected-to-normal vision via contact lenses. They all had good stereopsis, for which they were tested via a standard TNO-test (Walraven, 1975). Their ages varied from 19 to 27 years. The subjects performed three experimental sessions. The first was considered to be a training session. One of the subjects was rejected after the second session, because she showed a number of mirrored settings (toward her, instead of away from her) in the condition without the context in this session.

\section{Apparatus}

We used a virtual 3-D set-up. The stimuli were generated on a Silicon Graphics Indy computer. This computer generates $120 \mathrm{im}$ ages per second and draws the projection of a 3-D image for each eye onto the screen, alternately one for the right eye and one for the left eye. The subjects wore LCD goggles that closed one or the other eye synchronously with the images presented on the computer screen. The goggles work best for red images.

The computer screen consisted of $1,280 \times 1,024$ pixels. The pixels were $0.27 \times 0.27 \mathrm{~mm}^{2}$. We used anti-aliasing commands to increase the resolution. A circular band of black cardboard was placed around the computer screen, in order to exclude possible reference directions being indicated by the edges of the screen.

\section{Stimuli}

The subjects could always see a red pointer and a red target. The pointer was a wire-frame figure that consisted of a $4-\mathrm{cm}$ line and a ring with a diameter of $3.2 \mathrm{~cm}$. The plane of the ring was perpendicular to the line segment, and it was located around one of the endpoints of the line. The target consisted of a dot (1 pixel) with two rings extending in 3-D drawn around it. The two rings had a diameter of $1.2 \mathrm{~cm}$ and met each other directly above and below the dot. The target and the pointer were located in a horizontal plane at eye level. The midpoint between the target and the pointer was located on the computer screen and was $120 \mathrm{~cm}$ in front of the cyclopean eye of the subject. The pointer could appear at eight possible locations (see the ellipses in Figure 1). The target appeared at the opposite side (of the dashed lines in Figure 1). The distance between the rotation point of the pointer and the target was $20 \mathrm{~cm}\left(7.3^{\circ}\right.$ or $8.6^{\circ}$ visual angle, depending on the orientation of the connecting line between the target and the pointer).

We performed three experiments: one without any context and two with a context. In all the experiments, the possible locations of the pointer and the target remained constant, whereas the context, if present, was varied. The context consisted of two flat planes. Figure 2 shows how these planes were varied for one possible combination of pointer and target locations for the two experiments with context. In the rotation experiment (Figure 2A), the two planes were always perpendicular to each other. The orientation of the two 




Figure 1. A scaled top view of the eight possible locations at which the pointer and the target could appear (at the ellipses). Given the location of the pointer, the target appeared at the opposite side of the dashed line. For each combination of pointer and target, its mirror image in the frontoparallel plane or the midsagittal plane also exists. The connecting straight line between the target and the pointer could make an angle of $25^{\circ}$ or $40^{\circ}$ with the frontoparallel plane. The midpoint of the connecting line between the target and the pointer lay $120 \mathrm{~cm}$ in front of the cyclopean eye of the subject and on the computer screen. The symbols near the ellipses are those used in Figures 4 and 5 for the corresponding location of the pointer.

planes with respect to the subject's frontoparallel plane was varied in six steps from $0^{\circ} / 90^{\circ}$ to $45^{\circ} / 45^{\circ}$, which we shall call rotations $0-45$, respectively. In the angle experiment (Figure $2 \mathrm{~B}$ ), the planes were always oriented symmetrically with respect to the subject's frontoparallel plane. Here, the dihedral angle was varied in six equal steps from $90^{\circ}$ to $40^{\circ}$ (i.e., with respect to the subject's frontoparallelplane, they varied from $45^{\circ} / 45^{\circ}$ to $70^{\circ} / 70^{\circ}$ ).

The contextual planes met in one common vertical line (like two walls of a room). They consisted of a blue grid ${ }^{1}$ of horizontal and vertical lines, indicating squares of $2.5 \times 2.5 \mathrm{~cm}^{2}$. The grid extended from 2.5 squares above to 2.5 squares below the target and the pointer and from the intersection line between the planes to about 1.5-2.5 squares beyond the target and the pointer. The planes were at least 4 squares wide. Two $5-\mathrm{cm}$-long blue vertical lines were drawn, one through the target and one through the rotation point of the pointer, to emphasize that the target and the pointer were located in the contextual planes.

\section{Procedure}

The subject's task was to orient the pointer so that it aimed at the center of the target. The orientation of the pointer in the horizontal plane could be manipulated by pressing the arrow keys on the keyboard. The subjects could use as much time as they needed. We recorded the final orientation of the pointer.

Our subjects completed three experimental sessions. The first session served as a training session and was identical to the third session. Each of the sessions started with the experiment in which no contextual planes were present, followed by one of the possible two experiments with contextual planes.

The order of the experiments with the contextual planes (rotation or angle), the order of the stimuli, and the starting orientation of the pointer were randomized. The pointer appeared at an orientation between plus and minus $25^{\circ}$ around the veridical orientation and could appear at one out of eight different locations, with the target's appearing opposite (see Figure 1). Each possible location of the pointer could appear with six different contexts (see above), resulting in 48 stimuli per experiment with contextual planes. All the stimuli were presented five times. The experiment was divided into blocks of 15-30 min, followed by a short break of a few minutes, to keep the subjects alert. One complete experimental session took about $2 \mathrm{~h}$.

\begin{abstract}
Analysis
We recorded the final orientation of the pointer at which the subjects judged the pointer to aim at the target. The pointer orientation was defined to be $-90^{\circ}$ when oriented perpendicular to the frontoparallel plane pointing toward the subject, $0^{\circ}$ when oriented in the frontoparallel plane of the subject, and $+90^{\circ}$ when oriented perpendicular to the frontoparallel-plane pointing away from the subject. Thus, a pointer orientation mirrored in the midsagittal plane had the same value. This was done because a previous study had shown that exocentric pointing is symmetrical with respect to the midsagittal plane (Schoumans \& Denier van der Gon, 1999).

For each stimulus (a possible combination of the pointer and the target locations in a certain context) and for each subject, we calculated the mean set orientation of the pointer and the standard deviation from the mean. To demonstrate an influence of contextual surfaces, we compared the set orientations of the pointer in conditions with and without contextual planes. We defined a change in the set orientation of the pointer as the absolute mean value in the condition with the contextual planes minus the absolute mean value in the condition without contextual planes, where both were measured within the same experimental session. Thus, a positive value of change means that the subject oriented the pointer more steeply (i.e., at a larger slant) in the presence of the contextual planes, as compared with when those planes were not present.
\end{abstract}

\section{RESULTS}

The experiment without contextual planes was measured twice, once before each experiment with a context. A comparison of the two measurements shows that the set pointer orientations reproduced well. Figure 3 shows the mean orientation of the pointer in the condition without a context (measured before the rotation experiment) as a function of the veridical orientation of the pointer. Each subject is represented by a different symbol. Without context, subjects M.L., P.M., and R.O. tended to set the orientation of the pointer at too large a slant; C.N. tends to do the opposite. The standard deviation of the means (calculated over five repetitions) is $3^{\circ}$ for subjects M.L., P.M., and R.O. and $4.5^{\circ}$ for subject C.N. on average, over all the pointer locations. Therefore, the mean orientations in stimuli, which are mirror images of each other in the midsagittal plane, are generally equal within the errors of the means. This is consistent with the earlier Schoumans and Denier van der Gon (1999) results and can be seen in Figure 3 by comparing two equal symbols at each veridical orientation.

Figure 4 shows the results of the rotation experiment. For all the subjects, the change in set pointer orientation with respect to the condition without a context is plotted as a function of the rotation of the planes. The rotation is defined as the smallest angle between one of the planes and the frontoparallel plane. Thus, a $45^{\circ}$ rotation of the 


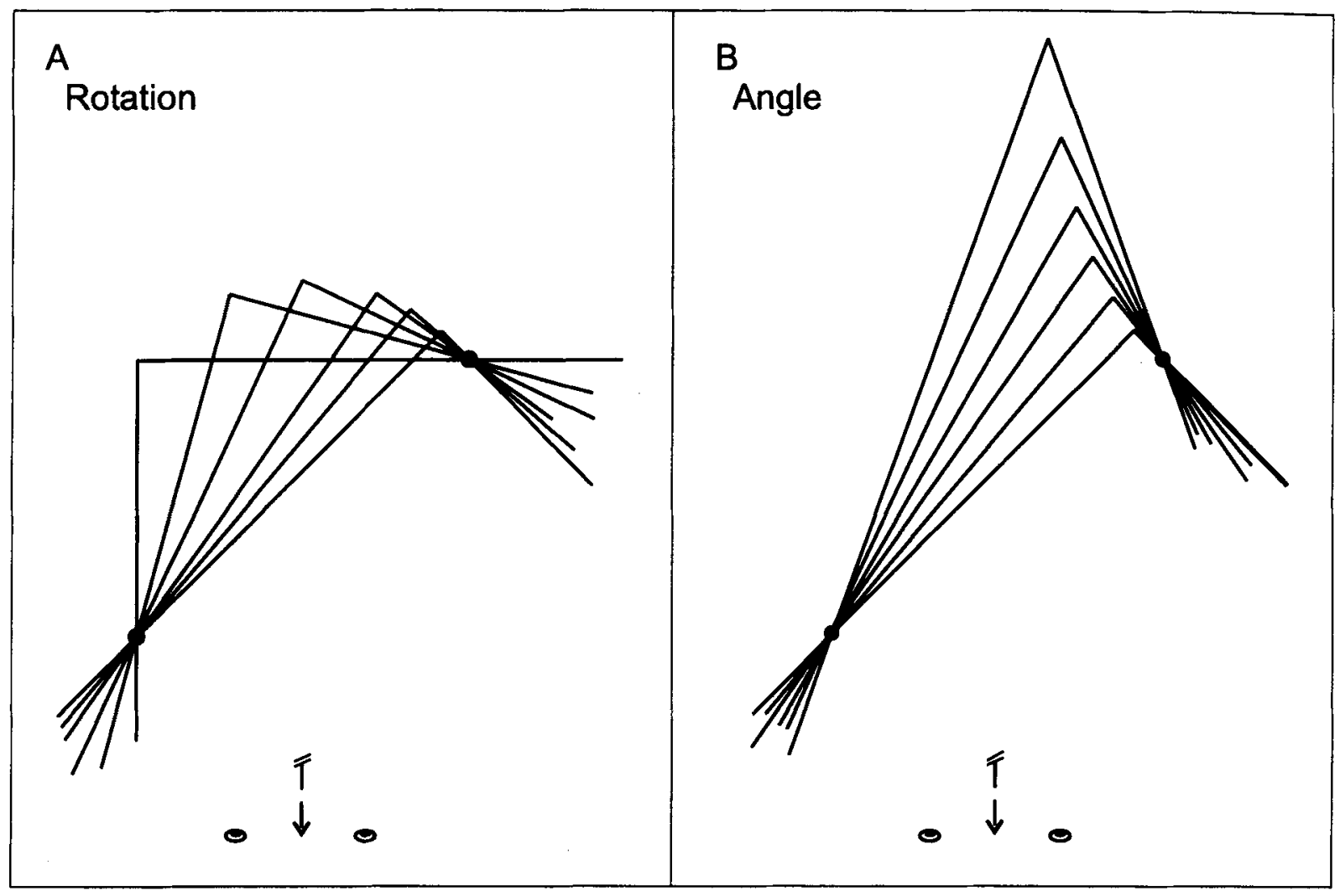

Figure 2. Illustration of the variations in contextual planes, as used in the experiments with context for a possible combination of pointer (one of the dots) and target (the other dot) locations. In these experiments with a context, each possible combination of pointer and target locations could appear with one of six possible contexts. Panel $A$ illustrates the rotation experiment. In this experiment, the contextual plane through the target and the contextual plane through the pointer intersected at a dihedral angle of $90^{\circ}$. The orientation of the contextual planes with respect to the subject was varied. This is indicated by a value of rotation that could be $0^{\circ}, 15^{\circ}, 25^{\circ}, 35^{\circ}, 40^{\circ}$, or $45^{\circ}$, which equals the smallest angle between one of the planes and the frontoparallel plane. Panel $B$ illustrates the angle experiment. In this experiment, the contextual plane through the pointer and the one through the target made the same angle with the fronto parallel plane. The dihedral angle between the planes was varied and could be $90^{\circ}, 80^{\circ}, 70^{\circ}, 60^{\circ}, 50^{\circ}$, or $40^{\circ}$. Note that, in both experiments, the condition exists with a dihedral angle of $90^{\circ}$ and a rotation of $45^{\circ}$. Also note that, to keep the pointer and the target located in the planes, the point where the planes met had to be translated, and the lengths of the planes had to be adjusted.

planes means that both planes were oriented symmetrically with respect to the frontoparallel plane of the subject. (See Figure 2A for an illustration of the variations in context.) Each possible location of the pointer is indicated by a different symbol (see Figure 1). Similarly shaped symbols represent stimuli that are each other's mirror image in the midsagittal plane. Dashed lines connect the symbols representing pointer locations that are located in front of the target.

Figure 4 shows that the presence of the planes influenced the set pointer orientation systematically. This influence was reflected in changes in the set pointer orientation, ranging between $+25^{\circ}$ and $-20^{\circ}$. Moreover, the changes varied systematically with the variation in the orientation of the planes with respect to the subject. There was a global trend for all the subjects to set the pointer at a larger slant when one of the planes lay in a frontoparallel plane and at a smaller slant when the planes were oriented symmetrically to the frontoparallel plane. The trend, on average, over pointer locations has a gradient of $0.33^{\circ}, 0.32^{\circ}, 0.24^{\circ}$, and $0.34^{\circ}$ per degree of rotation for subjects C.N., M.L., P.M., and R.O., respectively. This trend is significant for each of the subjects [analysis of variance (ANOVA) per subject, with the rotation entered as covariate first: $N=240$ ( 5 repetitions $\times 8$ pointer locations $\times 6$ contexts), $F(1,227)>271, p<.0005]$.

Figure 5 is similar to Figure 4, except that it shows the change in set pointer orientation as a function of the dihedral angle between the planes (see Figure 2B for an illustration of context variation). For 3 of our 4 subjects (M.L., P.M., and R.O.), there was a clear change in the set orientation of the pointer between the conditions with and without the contextual planes. Furthermore, there seems to have been a small global trend to set the pointer at a larger slant when the dihedral angle became smaller. This trend, on average, over pointer locations, has a gradient of $0.096^{\circ}, 0.098^{\circ}, 0.016^{\circ}, 0.072^{\circ}$ per degree of angle for subjects C.N., M.L., P.M., and R.O., respec- 


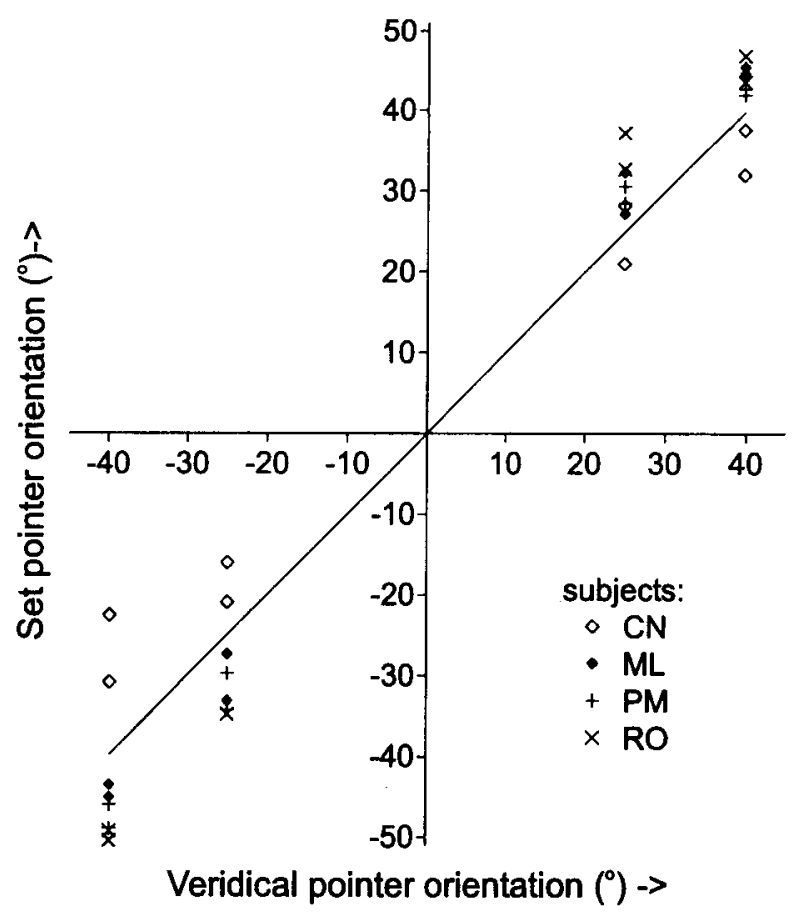

Figure 3. Exocentric pointing without any context. The mean orientation of the pointer is plotted, for each of its possible locations, as a function of the veridical orientation of the pointer. Each subject is indicated with a different symbol. Two identical symbols at one veridical orientation belong to two pointer (and target) locations that are symmetrical with respect to the midsagittalplane. The standard deviations are approximately $3^{\circ}$ for subjects M.L., P.M., and R.O. and approximately $4^{\circ}$ for subject C.N.

tively. This trend is significant for each of the subjects [ANOVA per subject, with the angle entered as covariate first: $N=240$ ( 5 repetitions $\times 8$ pointer locations $\times 6$ contexts), $F(1,227)>31, p<.0005]$, except for subject P.M. $(F=1.2, p=.28)$.

The condition in the angle experiment in which the angle was $90^{\circ}$ equals the condition in the rotation experiment when the rotation was $45^{\circ}$. A comparison of this condition in Figures 4 and 5 shows a reasonable reproduction of the measured change in the set pointer. To be more precise, the mean set orientations of the pointer are not significantly different in 27 of the 32 cases (twotailed $t$ test, $d f=4, p<.05$ ).

As can be seen in both Figures 4 and 5, there are noticeable differences between the subjects. The most apparent difference between the subjects is that their plots are shifted with respect to each other. This is mainly due to the differences between subjects in set pointer orientation in the experiment without a context. The deviations from the veridical in the experiment with a context lie in the same range of $35^{\circ}$ for all the subjects, between $20^{\circ}$ too large a slant and $15^{\circ}$ too small a slant. In other words, our subjects' set orientations of the pointer resemble one anothers' more closely in the conditions with a context.
An ANOVA over all subjects also showed significant interactions between subjects and pointer locations, as can also be seen in Figures 4 and 5. For example, subject R.O. shows an asymmetry in his settings between the pointers pointing forward and those pointing backward toward the target. In the rotation experiment, the trend is less strong for the pointers pointing backward. This results in a segregation between the two groups at a rotation of the planes of $45^{\circ}$, which persists throughout the angle experiment. In contrast to this, the settings of subject M.L. clearly do not show such an asymmetry between these two groups. As another example, the top line in the graph for subject C.N. in Figure 4 has a noticeable displacement in relation to the other lines. Such a displacement may be due to the variance in the orientation set for that pointer location in the condition without a context, which results in a relatively large standard error of the mean, since it was calculated over only five repetitions. This error displaces all the set orientations in the conditions with a context for that corresponding pointer location by the same constant. In this case, however, this displacement also appears in Figure 5, because the mean set pointer orientation in the experiment without the context is reproduced.

Besides the influence of context on the average set orientation of the pointers, a context could also influence consistency in pointing. We compared the standard deviations in the conditions with and without the contextual planes by performing an independent samples $t$ test for each of the subjects. Only for 1 subject, C.N., could we reject the hypothesis that the standard deviations were the same with and without the planes $[F(1,58)=$ $16.7, p<.001]$. The standard deviations in her settings were, on average, $1.5^{\circ}$ larger in the experiment without context, as compared with the condition with a context. For the other 3 subjects, the standard deviations were not statistically different $(p=.81, .96$, and .96 for subjects M.L., P.M., and R.O., respectively).

We also checked whether the standard deviations changed systematically with the variations in the contexts. For both experiments with a context, the measured standard deviations were fitted with a linear regression line as a function of the variations in the planes. None of these regressions deviated significantly from zero $[N=48$, 8 pointer locations $\times 6$ contexts, $F(1,46)<2.8$; for subjects C.N., M.L., P.M., and R.O., respectively, $p=.19$, $.44, .10$, and .09 in the rotation experiment, and $p=.50$, $.64, .36$, and .47 in the angle experiment]. The measured standard deviations in the experiments with a context were about $3^{\circ}$ for each of the subjects.

\section{POSSIBLE ORIGINS OF INFLUENCE}

The results have shown that contextual planes have a significant influence on the mean exocentric pointed direction. The experiments were designed to demonstrate the existence of an influence, not to find out the precise 

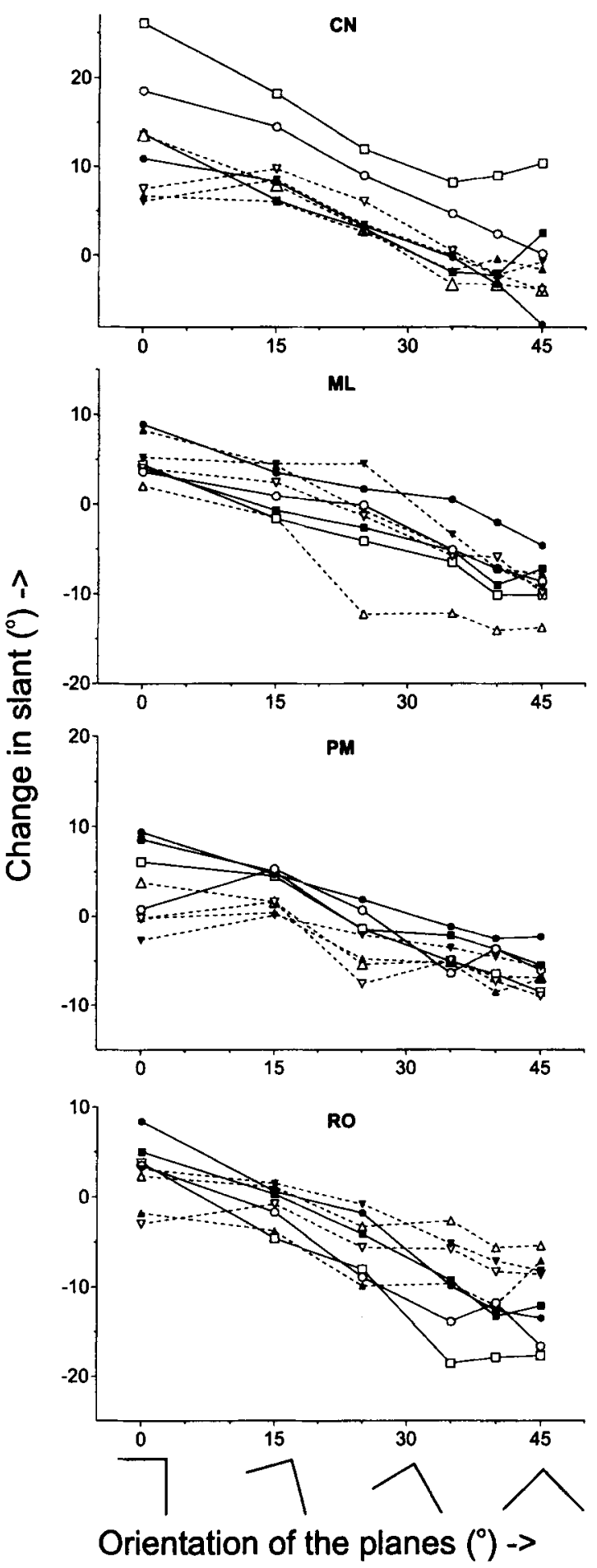

Figure 4. A graph drawn for each subject shows the change in orientation of the pointer as a function of the orientation of the planes with respect to the subject. The two planes are always perpendicular. $A 0^{\circ}$ orientation of the planes means that one of the planes lay in a frontoparallel plane. $A 4^{\circ}$ orientation of the planes means that the two planes were oriented symmetrically with respect to the frontoparallel plane. The change in orientation of the pointer is defined as its absolute value with contextual planes minus its absolute value without the contextual planes. In other words, a positive value for this change means an increase in the slant of the pointer as a result of the contextual planes. A different symbol indicates a different location of the pointer (see Figure 1). nature of this influence. Nevertheless, we can make several observations about the possible origins of the demonstrated influence.

First of all, we can exclude several strong candidates for the role of a single determining factor underlying the results of both our experiments with a context. For example, the orientation of the contextual plane through the target with respect to the subject changed over a range of $45^{\circ}$ in the rotation experiment and over a range of $25^{\circ}$ in the angle experiment (see Figure 2). If this was the only determining factor in our results, we should have found a trend in the angle experiment that was about half as large as the trend in the rotation experiment. This is not what we observed. The same line of reasoning can exclude the orientation of the plane through the pointer and the angle between the pointer orientation and the plane in which it lay. To keep the pointer and the target in the planes, we also had to vary other parameters besides the rotation and the dihedral angle. These parameters can also be excluded as single determining factors. The range of depth and disparities over which the planes extended and, thus, also the length of the planes that we had to present, as well as the area defined by the target, the pointer, and the intersection line of the planes, varied most in the angle experiment (see Figure 2), where we found the smallest effects. The eccentricity of the intersection line of the planes varied from the right hemifield to the left hemifield for one possible combination of the pointer and the target locations (see Figure 2). If this were the sole determining factor, the trend in the rotation experiment should have been symmetrical with respect to the condition in which this line lies straight in front of the cyclopean eye of the subject (at a rotation of $25^{\circ}$ ). Again, this was not what we observed. In conclusion, it would seem likely that there is more than one factor determining the results of the measurements with the contexts.

Second, there is no apparent link between the demonstrated influence of context on perceived spatial relations and the specific influence of context demonstrated in other types of experiments reported in the literature. The experiments mentioned in the introduction suggest an influence of context on perceived orientation of objects and perceived depth differences. Those results are often explained in terms of reference frames for the horizontal and frontoparallel planes (Howard, 1982; Kumar \& Glaser, 1992). In depth contrast experiments and rod frame experiments, a redefinition of the frontoparallel plane or the horizontal plane is presumed to take place as the largest visible plane or frame is rotated. This would indicate a simple rotation of visual space in relation to physical space. However, such a rotation (or any other affine transformation) does not result in systematic errors in the indicated exocentric direction. The observed influence on the indicated direction needs a more complex explanation.

Finally, there are several possible directions in which to search for an origin of the influence of the contextual planes on perceived spatial relations. The contextual planes could provide more structure for visual space. They could distort visual space. They could provide planes of 


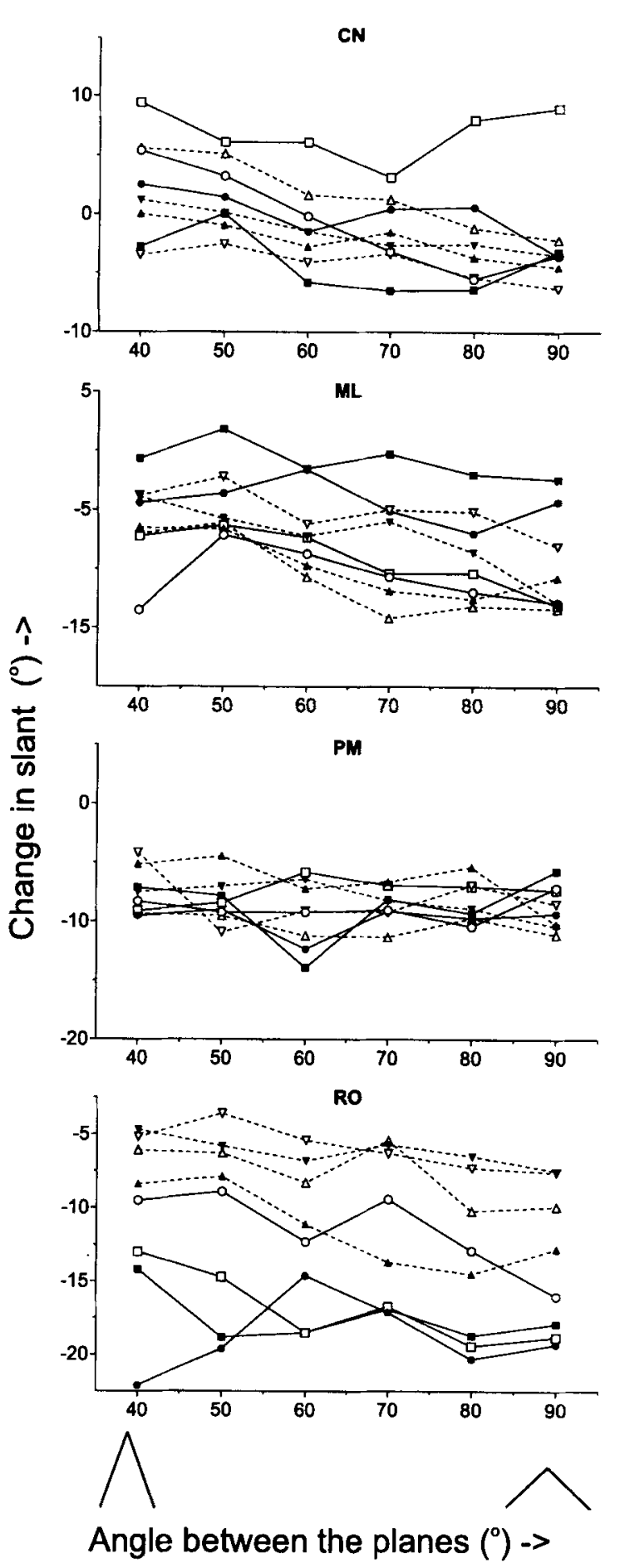

Figure 5. A graph drawn for each subject plots the change in set pointer orientation as a function of the dihedral angle between the planes. The two planes were always oriented symmetrically with respect to the frontoparallel plane.

reference. Or the perceived spatial relations could be codetermined by perception of the shape of the planes. The first two options do not seem likely. The first would predict the smallest standard deviations in the conditions with the contextual planes (see the General Discussion and Conclusions section), which we did not observe. The second option is not likely, because a distortion of visual space would lead to the perception of a straight line in space as curved. In other words, two stimuli with their pointer and target locations interchanged should then lead to set orientations of the pointers so that they both point in front or behind the connecting line. This was generally not what we observed. The third option, the planes' providing some sort of reference, might explain why our subjects' set pointer orientations resemble each other more closely when a context is present than when one is not. On the possible influence of perception of the shape of the planes, we can only speculate. In summary, finding out the precise nature of the influence of context will require diverse, separate studies.

\section{GENERAL DISCUSSION AND CONCLUSIONS}

We have shown that the addition of a context in the visual scene has a significant influence on the indicated exocentric direction. Therefore, we conclude that exocentric pointing depends on the contents of the visual scene. This important conclusion is strengthened by the fact that the changes in the indicated exocentric direction vary systematically with the main variations in the contextual planes: the orientation of the two contextual planes with respect to the subject and, to a lesser extent, the angle between the two contextual planes.

In contrast to the significant influence of contextual planes on the measured mean indicated exocentric direction, no such influence was found on the measured standard deviations. The standard deviations did not even change between the conditions with and without the contextual planes for 3 of our 4 subjects. This is somewhat remarkable because, without the contextual planes, the visual scene is very barren and the contextual planes provide additional structure for the perceived space, which could help in localizing the target and the pointer. Therefore, we conclude that more structure in the visual field does not automatically lead to a more consistent perception of spatial relations.

How the influence of context works precisely will require additional research before better theories of visual space can be constructed (see the Possible Origins of Influence section). Gestalt theories have emphasized the importance of context but have only seldom given suggestions for the nature of the influence of context. Koffka (1935) mentions "the frameworks tendency to normality,"- that is, a horizontal ground plane and a vertical perpendicular on it. This does account for the results in the rod frame experiments, but it does not predict the significant and systematic changes in the indicated exocentric direction reported in this paper. The planes in our experiments were deliberately upright and consisted of a grid of squares, so that these planes would not give rise to illusions or suggest a false perspective cue. Nevertheless, we find that the average indicated exocentric direction changed significantly with the systematic variations in the planes. 
Our main conclusion remains clear: The stable perception of the 3-D space surrounding us depends on its contents. Thus, the transformation between perceived visual space and physical space cannot be constant. A constant transformation, however, is assumed by most models of visual space (e.g., Gilinsky, 1951; Luneburg, 1947; Wagner, 1985), since they assume that visual space has a metric and a geometry independent of its content. Thus, our results disprove all such models of visual space. New theories of visual space should include a role for context.

\section{REFERENCES}

AMEs, A. (1951). Visual perception and the rotation trapezoid window. Psychological Monographs, 65 (7, Whole No. 324), 1-32.

AMES, A. (1953). Reconsideration of the origin and nature of perception. In S. Ratner (Ed.), Vision and action. New Brunswick, NJ: Rutgers University Press.

Battro, A. M., di Pierro Netto, S., \& Rozestraten, R. J. A. (1975). Riemannian geometries of variable curvature in visual space: Visual alleys, horopters, and triangles in big open fields. Perception, 5, 9-23.

BLANK, A. A. (1961). Curvature of binocular visual space, an experiment. Journal of the Optical Society of America, 51, 335-339.

BLumenfeld, W. (1913). Untersuchungen über die Scheinbare Grösse in Sehräume. Zeitschrift für Psychologie, 65, 241-404.

Ellis, S. R., Smith, S., Grunwald, A., \& MCGreevy, M. W. (1991). Direction judgement error in computer generated displays and actual scenes. In S. R. Ellis (Ed.), Pictorial communication in virtual and real environments (Pt. IV, pp. 504-526). London: Taylor \& Francis.

FoLEY, J. M. (1964). Desarguan property of visual space. Journal of the Optical Society of America A, 54, 648-692.

FolEY, J. M. (1991). Binocular space perception. In D. Regan (Ed.), Vision and visual dysfunction: Vol. 9. Binocular vision (pp. 75-92). Houndsmills, U.K.: MacMillan.

FOLEY, J. M., \& RiCHARDS, W. (1972). Effects of voluntary eye movement and convergence on the binocular appreciation of depth. Perception \& Psychophysics, 11, 423-427.

GiBson, J. J. (1950). The perception of the visual world. Cambridge, MA: Riverside.

Gilinsky, A. (1951). Perceived size and distance in visual space. Psychological Review, 58, 460-482.

GoGEL, W. C. (1984). The role of perceptual interrelations in figural synthesis. In P. C. Dodwell \& T. Caelli (Eds.), Figural synthesis. Hillsdale, NJ: Erlbaum.

Gogel, W. C., \& Mershon, D. H. (1977). Local autonomy in visual space. Scandinavian Journal of Psychology, 18, 237-250.
Helmholtz, H. (1962). Helmholtz's treatise on physiological optics (Translation of 3rd German edition; J. P. C. Southall, Ed.). New York: Dover. (Original work published 1866)

HowARD, I. P. (1982). Human visual orientation. Chister, U.K.: Wiley. InDOW, T., \& WatanaBe, T. (1984). Parallel-alleys and distance-alleys on horopter plane in the dark. Perception, 13, 165-182.

KoENDERINK, J. J., \& VAN DOORN, A. J. (1998). Exocentric pointing. In L. R. Harris \& M. Jankin (Eds.), Vision and action (pp. 295-313). Cambridge: Cambridge University Press.

KofFKA, K. (1935). Principles of Gestalt psychology. New York: Harcourt, Brace \& World

Kumar, T., \& Glaser, D. A. (1992). Influence of remote objects on local depth perception. Vision Research, 31, 1687-1699.

LUNEBURG, R. K. (1947). Mathematical analysis of binocular vision. Princeton, NJ: Princeton University Press.

Mitchison, G. J., \& Westheimer, G. (1984). The perception of depth in simple figures. Vision Research, 24, 1063-1073.

PirenNe, M. H. (1970). Optics, painting and photography. Cambridge: Cambridge University Press.

Schoumans, N., \& Denier VAN der Gon, J. J. (1999). Exocentric pointing in three-dimensional space. Perception, 28, 1265-1280. Tolansky, T. (1964). Optical illusions. Oxford: Pergamon.

VAN Ee, R., \& ERKELENS, C. J. (1996). Anisotropy in Werner's binocular depth-contrast effect. Vision Research, 36, 2253-2262.

WAGNer, M. (1985). The metric of visual space. Perception \& Psychophysics, 38, 483-495.

WALRAVEN, J. (1975). Amblyopia screening with random-dot stereogrammes. American Journal of Ophthalmology, 8, 178-182.

WERNER, H. (1938). Binocular depth contrast and the conditions of the binocular field. American Journal of Psychology, 51, 489-497.

WiTKIN, H. A., \& ASCH, S. E. (1948). Studies in space orientation: IV. Further experiments on perception of the up-right with displaced visual fields. Journal of Experimental Psychology, 38, 762-782.

\section{NOTE}

1. The color of the grid was chosen to be different from the color of the target and the pointer in order to prevent ambiguities, such as possible disparity mismatches between the endpoint of the pointer and a line in the grid. However, a difference in color can lead to differences in perceived depth (Helmholtz, 1866/1962). In our experiments, this could not influence the results, since the color difference between the grid and the target and pointer was constant throughout the experiments with the contextual planes, and the pointer and the target were red in both experiments, with and without context.

(Manuscript received June 23, 1998; revision accepted for publication March 29, 1999.) 